\section{Topographical distribution of choroidal naevi in the ocular fundus}

\begin{abstract}
Objective To analyse the topographical distribution of choroidal naevi and to visualise their location in the ocular fundus.
\end{abstract}

Methods Data on the size and location of 210 choroidal naevi were converted into a database of two-dimensional retinal charts by means of computer-drawing software. The geometric centre of each lesion was entered into corresponding sectors of the retinal chart. The location of the naevi was computationally visualised by merging the fundus drawings and displaying the number of overlapping lesions on colour-coded contour maps.

Results Five naevi were located exactly between two fundus sectors, and were therefore excluded from the distribution analysis. Ten naevi $(5 \%)$ were located anterior and $195(95 \%)$ posterior to the equator. A total of 104 naevi $(51 \%)$ were located in the temporal and $101(49 \%)$ in the nasal hemisphere, and the distribution between the superior and inferior hemisphere was 104 $(51 \%)$ and $101(49 \%)$, respectively. The distribution did not differ significantly between genders, age groups, or between right and left eyes. More naevi with a diameter of $>3 \mathrm{~mm}$ were located in the temporal hemisphere $(P=0.0004)$ and anterior to the equator $(P=0.006)$ compared with those with a diameter of $\leqslant 3 \mathrm{~mm}$. A similar distribution was found for naevi with overlying drusen. Conclusions Choroidal naevi are uniformly concentrated in the centre of the posterior pole without any significant nasotemporal or superoinferior asymmetry. However, large naevi occur significantly more often in the temporal hemisphere and more anteriorly compared with small lesions.

Eye (2009) 23, 1685-1690; doi:10.1038/eye.2008.350; published online 28 November 2008
J Krohn ${ }^{1,2}$, T Frøystein ${ }^{3}$ and O Dahl ${ }^{3,4}$

Keywords: choroidal naevus; topography;

distribution; location; imaging

Introduction

Choroidal naevi are common ocular lesions with reported prevalence rates ranging from 1.9 to $6.5 \%$ and from 9 to $11 \%$ in population-based studies and autopsy series, respectively. ${ }^{1-4}$ A choroidal naevus usually presents as a flat or slightly elevated, brown to slate-grey lesion in the posterior fundus. The margins are typically indistinct, and often there are overlying drusen. Choroidal naevi may lead to visual loss and visual field defects, 5,6 but they are usually asymptomatic and incidentally found during routine examinations. The clinical significance of choroidal naevi lies in the difficulties in distinguishing them from small malignant melanomas, and in their potential risk for malignant transformation. ${ }^{7-9}$

Although many reports on choroidal naevi include some information about their fundus distribution, accurate data on this topic are scarce. The main objectives of this study were to analyse the topographical distribution of choroidal naevi and to present a method for visualising their location in the ocular fundus. We also wanted to compare these results with earlier studies on uveal melanoma location.

Materials and methods

\section{Patients}

The medical records of all patients with the diagnosis of choroidal naevus evaluated at the Department of Ophthalmology, Haukeland University Hospital, from 1993 through 2007 were reviewed. Only patients with records containing detailed information about the size and location of the naevi, confirmed by fundus photographs and/or fundus drawings, were included in the study. The clinical parameters
${ }^{1}$ Department of Clinical Medicine, Section of Ophthalmology, University of Bergen, Bergen, Norway

${ }^{2}$ Department of

Ophthalmology, Haukeland University Hospital, Bergen, Norway

${ }^{3}$ Department of Oncology and Medical Physics, Haukeland University Hospital, Bergen, Norway

${ }^{4}$ Institute of Medicine, Section for Oncology, University of Bergen, Bergen, Norway

Correspondence: J Krohn, Department of Ophthalmology, Haukeland University Hospital,

N-5021 Bergen,

Norway

Tel: +47559 741 15;

Fax: + 4755974143

E-mail: jorgen.krohn@ helse-bergen.no

Received: 9 June 2008 Accepted in revised form: 21 October 2008 Published online: 28 November 2008 
extracted from the records included gender, age at presentation, ocular symptoms, causes of referral, medical history, and follow-up time. The study was approved by the Norwegian Social Science Data Services, and followed the official ethical regulations for clinical research.

\section{Naevus data}

Data regarding morphologic features of the choroidal naevi were obtained from the written records and by examination of available fundus photographs, fundus drawings, fluorescein angiograms, and ultrasound A- and B-scans. Recorded data included the largest basal diameter and distance of the nearest naevus margin to the foveola and optic disc margin, which were estimated clinically by using the horizontal diameter of the optic disc as a reference of $1.75 \mathrm{~mm} .{ }^{10,11}$ The naevi were defined as small for lesions with a diameter of $\leqslant 3 \mathrm{~mm}$, and large for those with a diameter of $>3 \mathrm{~mm}$. For patients who were examined by ultrasonography, the thickness of the naevus was registered. In addition, naevus characteristics were recorded regarding shape, colour, and the presence of drusen, orange pigment, subretinal fluid, and choroidal neovascularisation. The location of the naevi was determined according to their geometric centre, which for the round and oval lesions corresponded to the midpoint of the largest diameter. For naevi with an asymmetrical shape, the location was assessed by judgement of the centremost point. The naevi were then categorised according to their anteroposterior location (relative to the equator) and to their meridional location (quadrants and hemispheres defined by a horizontal and vertical line passing through the fovea). Naevi that were located exactly between two sectors of the fundus were excluded from the distribution analysis.

\section{Fundus mapping}

On the basis of a thorough evaluation of all available fundus photographs and original fundus drawings, each naevus was drawn with azimuth equidistant projections on a standardised retinal drawing chart with a macular centre surrounded by circles representing the equator, ora serrata, and limbus. ${ }^{10,12}$ All drawings were done by one of the authors $(\mathrm{JK})$, and special care was taken to correct for circumferential distortion in the periphery when calculating retinal diagrams from the naevus parameters. ${ }^{13,14}$ Thereafter, the drawing tools of the computer software PowerPoint (Microsoft Corp., Redmond, WA, USA) were used to convert all the drawings into a database of identical two-dimensional retinal charts of right eyes. On the basis of these drawings, the computer softwares URT (Utah Raster Toolkit, University of Utah/University of Michigan, MI,
USA) and Excel (Microsoft Corp., Redmond, WA, USA) were used to automatically determine the geometric centre of each naevus and subsequently plot all the central points into one retinal chart. By means of the software packages URT, AVS (Advanced Visual Systems Inc., Waltham, MA, USA) and Matlab (the MathWorks Inc., Natick, MA, USA), the collection of digital fundus drawings was merged, filtered and finally converted into a contour map of the fundus, displaying the number of overlapping naevi with different colour codes. Separate contour maps were made for various subgroups of patients and naevi. Although the maximum number of overlapping lesions differed between these groups, each contour map was labelled with the same colour scale ranging from blue to red. The dark blue colour indicated areas without any naevi, and the dark red colour displayed the area with the maximum number of overlapping naevi.

\section{Statistical methods}

The $\chi^{2}$ goodness-of-fit test was used to analyse the distribution of the choroidal naevi under the null hypothesis that they were uniformly distributed in the ocular fundus. Because of possible sources of error when calculating the exact areas of the concentric zones anterior and posterior to the equator, a statistical analysis of the distribution of all naevi was only performed for their meridional location. This was carried out under the assumption that each quadrant or hemisphere includes an equal area of the choroidal sphere. However, for the comparison between two groups of patients or naevi with different characteristics (binary variables), the Fisher's exact test was used to analyse both the anteroposterior and the meridional distribution of the lesions. The Bonferroni correction for multiple comparisons was applied if necessary. For all tests, two-tailed $P$-values of $<0.05$ were considered to be statistically significant.

\section{Results \\ Patient and naevus characteristics}

We identified 202 patients (119 women and 83 men) with choroidal naevi that met our criteria for inclusion into the study. Eighty patients (40\%) had been referred by ophthalmologists in practice, and $122(60 \%)$ had been diagnosed in our department on routine examination or during treatment for other eye diseases. The median age at the time of diagnosis was 67 years (range: 10-95 years). The right eye was involved in 106 patients, the left eye in 93 patients, and three patients had bilateral naevi. Five patients had two naevi in the same eye, leading to a total 
of 210 choroidal naevi eligible for analyses. Fifteen patients $(7 \%)$ had visual symptoms attributable to the naevus.

The median largest diameter was $2.6 \mathrm{~mm}$ (mean: $3.2 \mathrm{~mm}$; range: $0.6-9.6 \mathrm{~mm}) ; 114$ naevi $(54 \%)$ were classified as small ( $\leqslant 3 \mathrm{~mm}$ diameter) and $96(46 \%)$ as large ( $>3 \mathrm{~mm}$ diameter). The median distances from the nearest naevus margin to the foveola and to the optic disc were $3.5 \mathrm{~mm}$ (range: $0-17.0 \mathrm{~mm}$ ) and $4.0 \mathrm{~mm}$ (range: 0-17.0 mm), respectively. Among 53 naevi examined by ultrasonography, the median thickness was $0 \mathrm{~mm}$ (mean: $0.7 \mathrm{~mm}$; range: $0-3.0 \mathrm{~mm})$. One naevus $(0.5 \%)$, with an initial diameter of $4.4 \mathrm{~mm}$ and a thickness of $1.0 \mathrm{~mm}$, located temporal to the macula, had documented evidence of growth and transformation into melanoma after 5 years of observation. Otherwise, no lesions were clinically noted to increase in size during a median follow-up time of 12 months (mean: 25 months; range: 0-167 months). The lesions were round in 123 of cases $(59 \%)$, oval in $64(30 \%)$, and irregular in $23(11 \%)$. A total of 196 naevi (93\%) were grey, six (3\%) were black, and eight $(4 \%)$ were graded as amelanotic. Overlying drusen were noted in 79 naevi (38\%), whereas no naevi displayed orange pigment or subretinal fluid. Four patients $(2 \%)$ had a choroidal neovascular membrane in association with a naevus in the macular or peripapillary region.

\section{Topographical fundus distribution}

The distribution of the central points for all the 210 naevi is illustrated in Figure 1. Five naevi were located exactly between two sectors of the fundus, and were therefore excluded from the distribution analysis. Ten naevi (5\%) were located anterior and $195(95 \%)$ posterior to the equator. A total of 104 naevi (51\%) were located in the temporal and $101(49 \%)$ naevi were in the nasal hemisphere $(P=0.83)$, and the distribution between the superior and inferior hemisphere was 104 (51\%) and $101(49 \%)$, respectively $(P=0.83)$.

The number of naevi and their topographical distribution according to demographic and morphologic characteristics are presented in Table 1. The distribution did not differ significantly between genders, between patients in the age group of $\leqslant 60$ years and $>60$ years, or between right and left eyes. Significantly more naevi with a diameter of $>3 \mathrm{~mm}$ were located in the temporal hemisphere $(P=0.0004)$ and anterior to the equator $(P=0.006)$ compared with those with a diameter $\leqslant 3 \mathrm{~mm}$. Similarly, more naevi with overlying drusen were located in the temporal hemisphere compared with those without any drusen $(P=0.0005)$. After adjustment with Bonferroni corrections for multiple comparisons, these values were still statistically significant at the $P<0.05$

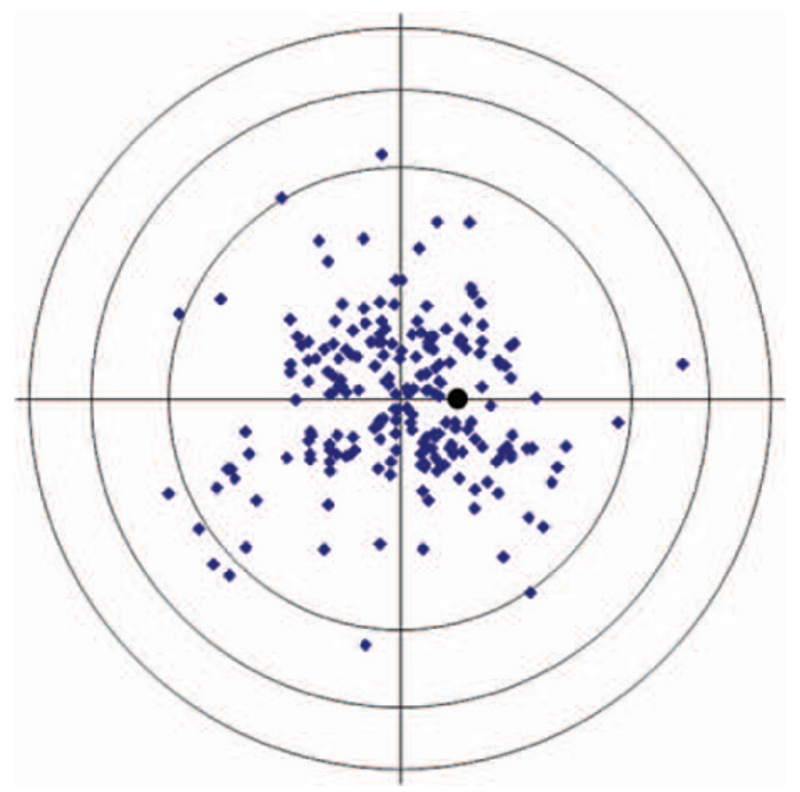

Figure 1 Central points of 210 choroidal naevi plotted on a retinal chart with a macular centre surrounded by circles representing the equator, ora serrata, and limbus. Note the uniform distribution between quadrants, and that the density of lesion centres gradually decreases with distance from the macula.

level. The predilection of large naevi to occur more temporally and anteriorly also remained statistically significant when the groups of referred and non-referred patients were analysed separately.

The location of the lesions, visualised by the computationally merged retinal charts, corresponded clearly with the abovementioned numerical distributions and revealed that choroidal naevi are uniformly concentrated in the centre of the posterior pole. The maximum number of overlapping naevi was 21 , and this area was centred in the macular and papillomacular regions (Figure 2). The merged charts also demonstrated that naevi classified as large were more likely to be located temporally and peripherally compared with small naevi (Figure 3a and b). Similarly, naevi with overlying drusen were located more temporally and peripherally than those without drusen.

\section{Discussion}

Earlier studies have shown that the majority of choroidal naevi are located posterior to the equator. ${ }^{4-6,8,15,16}$ Even when variations in data collection and the outlining of fundus quadrants are taken into account, reports on their meridional or quadrantic distribution are more conflicting. Choroidal naevi and small melanocytic lesions have been shown to be rather evenly distributed 
Table 1 Topographical distribution of 205 choroidal naevi in various fundus hemispheres and concentric zones, according to binary variables of patient and naevus characteristics

\begin{tabular}{|c|c|c|c|c|c|c|c|c|c|c|}
\hline \multirow[t]{2}{*}{ Binary variables } & \multirow[t]{2}{*}{ Number of naevi } & \multicolumn{6}{|c|}{ Hemispheres } & \multicolumn{3}{|c|}{ Concentric zones relative to the equator } \\
\hline & & $\mathrm{T}$ & $\mathrm{N}$ & P-value & $\mathrm{U}$ & $\mathrm{L}$ & P-value & Posterior & Anterior & P-value \\
\hline Female & 121 & 65 & 56 & 0.32 & 59 & 62 & 0.57 & 115 & 6 & 1.0 \\
\hline Male & 84 & 39 & 45 & & 45 & 39 & & 80 & 4 & \\
\hline$\leqslant 60$ years of age & 66 & 28 & 38 & 0.14 & 33 & 33 & 1.0 & 63 & 3 & 1.0 \\
\hline$>60$ years of age & 139 & 76 & 63 & & 71 & 68 & & 132 & 7 & \\
\hline Right eye & 105 & 57 & 48 & 0.33 & 58 & 47 & 0.21 & 100 & 5 & 1.0 \\
\hline Left eye & 100 & 47 & 53 & & 46 & 54 & & 95 & 5 & \\
\hline Largest basal diameter, $\leqslant 3 \mathrm{~mm}$ & 112 & 44 & 68 & $0.0004^{*}$ & 59 & 53 & 0.58 & 111 & 1 & $0.006^{*}$ \\
\hline Largest basal diameter, $>3 \mathrm{~mm}$ & 93 & 60 & 33 & & 45 & 48 & & 84 & 9 & \\
\hline No drusen & 127 & 52 & 75 & $0.0005^{*}$ & 66 & 61 & 0.67 & 124 & 3 & 0.05 \\
\hline Drusen & 78 & 52 & 26 & & 38 & 40 & & 71 & 7 & \\
\hline
\end{tabular}

$\mathrm{L}=$ lower; $\mathrm{N}=$ nasal; $\mathrm{T}=$ temporal; $\mathrm{U}=$ upper.

*These values remained statistically significant at the $P<0.05$ level after multiplicity correction on the basis of five different analyses, using the Bonferroni approach for multiple comparisons.

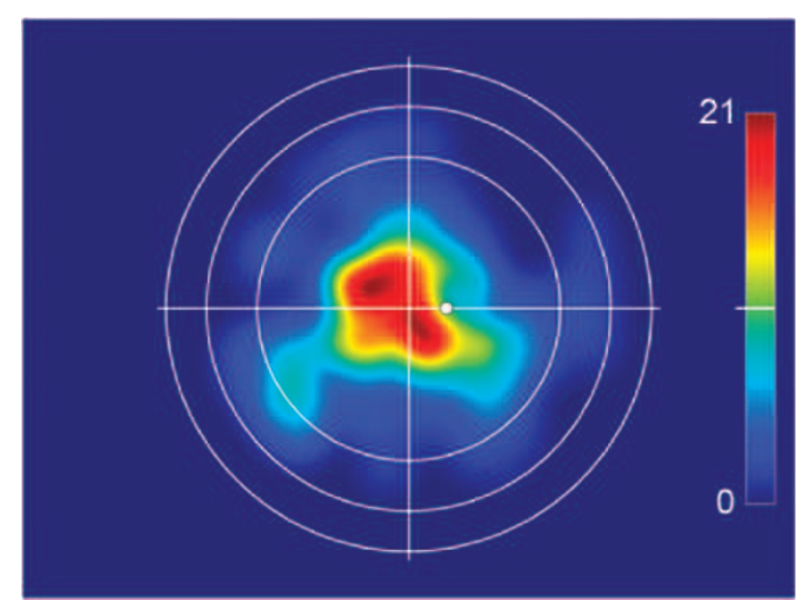

Figure 2 Merged fundus drawings showing the location of 210 choroidal naevi. The retinal chart has a macular centre surrounded by circles representing the equator, ora serrata, and limbus. The colours on the contour map indicate the number of overlapping naevi according to the colour scale bar. The top of the colour scale (dark red) represents the maximum number of overlapping lesions, and the bottom (dark blue) indicates no lesions. The maximum number of overlapping naevi is 21 .

in the posterior fundus, ${ }^{1,16,17}$ as well as having a predilection for both the nasal ${ }^{9}$ and temporal quadrants. ${ }^{4,8,15}$ In our study, the naevi were uniformly concentrated in the posterior pole. Both the numerical distribution and the merged fundus drawings demonstrated that choroidal naevi have a predilection for the macular region, without any significant nasotemporal or superoinferior asymmetry. A possible explanation for this finding could be that centrally located naevi, unlike peripheral lesions, are more easily observed during routine examinations. However, the preference for choroidal naevi to occur in the posterior pole has also been demonstrated in large autopsy series of healthy eyes, ${ }^{2,5}$ and a similar topographical distribution has been shown for posterior uveal melanoma. According to Li et al, ${ }^{12}$ the frequency of uveal melanoma occurrence is highest in the macular area and gradually decreases with distance from the macula to the ciliary body. The authors propose that this pattern correlates positively with the dose distribution of solar light on the retinal sphere, and their findings provide support for the controversial hypothesis that ultraviolet light exposure plays a role in the induction of uveal melanoma. Although several investigators have studied the relationship between sunlight exposure and the risk of uveal melanoma and some case-control studies may suggest an association, the existing data are incomplete and conflicting. ${ }^{18,19}$ Nevertheless, the relationship between sunlight exposure and the risk of cutaneous melanoma is well documented, ${ }^{20}$ and many reports have also shown an association between sunlight and the induction of cutaneous melanocytic naevi. ${ }^{21,22}$ The distribution of solar light on the retinal sphere may therefore account for the topographical fundus distribution of choroidal naevi found in this as well as in other studies, and we believe that this is the first study to ascertain this possibility. 

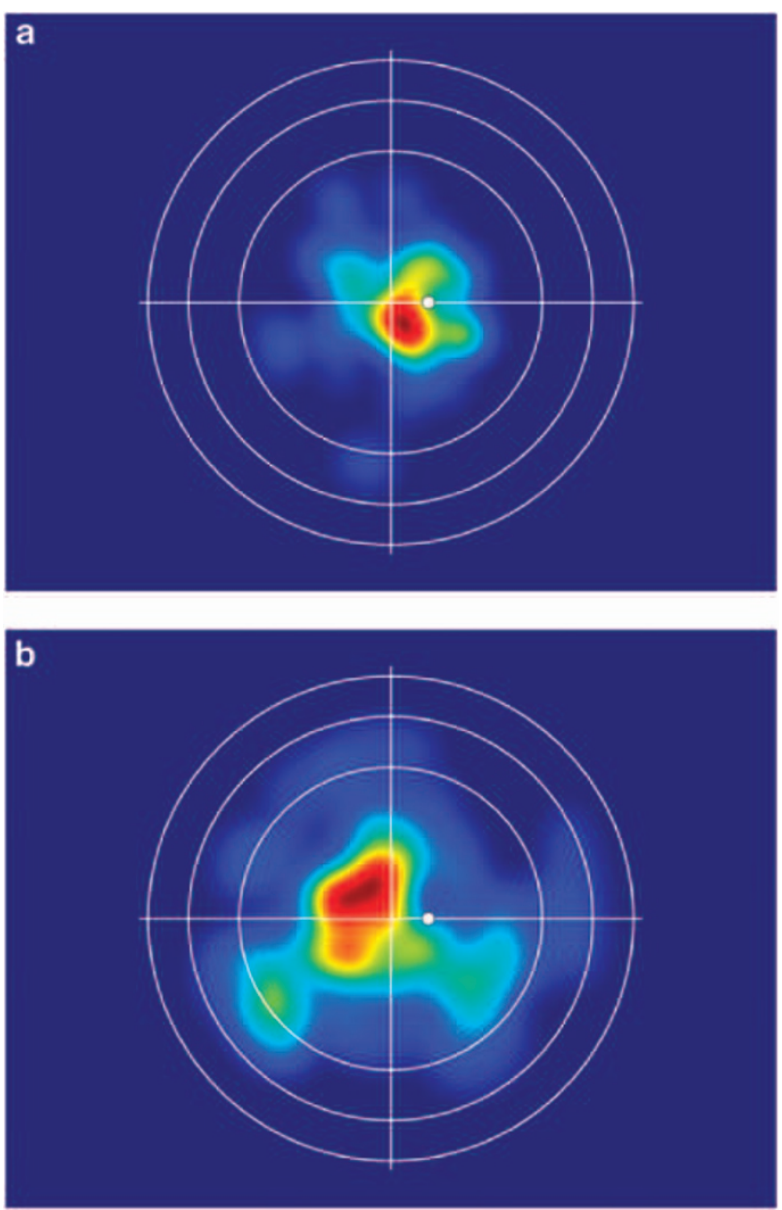

Figure 3 Merged fundus drawings showing the location of the naevi according to their size (largest diameter). The same colour scale as in Figure 2 is used for both images. Note that the maximum number of overlapping lesions (indicated by the dark red colour) differs between the images. (a) Small naevi ( $\leqslant 3 \mathrm{~mm}$ diameter); the maximum number of overlapping naevi is 13 . (b) Large naevi ( $>3 \mathrm{~mm}$ ); the maximum number of overlapping naevi is 14 .

The mean naevus diameter of $3.2 \mathrm{~mm}$ in our series is in accordance with earlier studies. In general, reports on naevus dimensions are highly dependent on study design, as larger naevi are found in clinic-based referral studies compared with population-based and autopsy series. In a clinic-based study by Shields et $a l,{ }^{23}$ the mean naevus diameter was $5.1 \mathrm{~mm}$, whereas the naevi in the population-based Blue Mountains Eye Study had a mean diameter of $1.25 \mathrm{~mm} .{ }^{4}$ In this study, $40 \%$ of the patients had been referred by ophthalmologists in practice and the remainder had been diagnosed in our department on routine examination or during treatment of other eye diseases.

When analysing subgroups of the naevi according to morphologic characteristics, we found that naevi measuring more than $3 \mathrm{~mm}$ in diameter occurred significantly more often in the temporal hemisphere and more anteriorly compared with smaller lesions. The anterior location could be related to the fact that small peripheral naevi are more easily ignored during ophthalmoscopy compared with larger lesions. On the other hand, most of the patients were recruited from our department, where all patients routinely undergo slitlamp biomicroscopy using a $90 \mathrm{D}$ precorneal lens or a diagnostic contact lens to examine the entire fundus through dilated pupils. An alternative explanation for both the peripheral and temporal location of large naevi could be site-specific differences of the choroid and retinal pigment epithelium affecting the proliferative potential and clinical characteristics of the naevi, similar to that proposed for the body-site distribution of melanocytic naevi. ${ }^{24-26}$ These studies have shown that naevus density is highest on sun-exposed body sites, such as the face, neck, and limbs, whereas larger naevi are most prevalent on the trunk, leading to the concept that melanocytic proliferation is modulated by anatomical location.

In a recent study, we have also shown that there is a significant nasotemporal asymmetry in the topographical distribution of uveal melanomas, and that the temporal hemisphere posterior to the equator is the preferential area of tumour occurrence and growth. ${ }^{27}$ With the assumption that naevus cells originate from melanocytes, it is tempting to speculate that there are developmental, site-specific variations between choroidal melanocytes, and that temporally located choroidal naevi have an increased potential of growth and malignant transformation compared with naevi located in the nasal fundus hemisphere. Some earlier studies have failed to show a relationship between the quadrantic location of small choroidal melanocytic lesions and their growth potential. ${ }^{9,28}$ However, we presume that our methods for analysing the topography of choroidal naevi provide more accurate data on this topic than those reported previously.

Similar to the distribution of large naevi, we found that naevi with overlying drusen had a more temporal and peripheral location compared with those without drusen. These observations are probably related, as the frequency of overlying drusen has been found to correlate with increasing naevus size as well as patient age., ${ }^{4,16}$

Because of the retrospective nature of the study and the incomplete follow-up, no firm conclusions can be drawn regarding the rate of malignant transformation of choroidal naevi. We found that one naevus evolved into melanoma after an observation period of 5 years. As choroidal naevi are much more frequent than uveal melanomas, most authors agree that such transformations are very rare. ${ }^{29,30}$ 
Our study has several potential limitations. First, the distribution analysis implies an isotropic growth of the naevi that is directed symmetrically from the geometric lesion centre. This is compensated for by the merged fundus drawings, which merely illustrate the frequency with which certain fundus areas are affected by naevi independent of their growth pattern. Second, the clinical estimation of naevus size, using the optic disc diameter as a reference, may theoretically lead to an overestimation of the most anteriorly located lesions. Other limitations include possible spherical distortions in the periphery when the naevus parameters were converted to the retinal charts, and the assumption that each hemisphere comprises an equal area of the fundus.

In summary, we found that choroidal naevi are uniformly concentrated in the centre of the posterior pole without any significant nasotemporal or superoinferior asymmetry. However, naevi with diameters larger than $3 \mathrm{~mm}$ have a predilection for the temporal hemisphere, similar to what has been reported for uveal melanoma location. Further studies are warranted to analyse whether temporal location of a choroidal melanocytic lesion can be considered as a possible risk factor for growth and malignant transformation.

\section{References}

1 Hale PN, Allen RA, Straatsma BR. Benign melanomas (nevi) of the choroid and ciliary body. Arch Ophthalmol 1965; 74: 532-538.

2 Naumann G. Pigmentierte Naevi der Aderhaut und des Glaskörpers. Adv Ophthalmol 1970; 23: 187-272.

3 Smith RS, Ganley JP. Ophthalmic survey of a community. 1. Abnormalities of the ocular fundus. Am J Ophthalmol 1972; 74: 1126-1130.

4 Sumich P, Mitchell P, Wang JJ. Choroidal nevi in a white population. The Blue Mountains Eye Study. Arch Ophthalmol 1998; 116: 645-650.

5 Naumann G, Hellner K, Naumann LR. Pigmented nevi of the choroid. Clinical study of secondary changes in the overlying tissues. Trans Am Acad Ophthalmol Otolaryngol 1971; 75: 110-123.

6 Gonder JR, Augsburger JJ, McCarthy EF, Shields JA. Visual loss associated with choroidal nevi. Ophthalmology 1982; 89: 961-965.

7 Rennie IG. Things that go bump in the light. The differential diagnosis of posterior uveal melanomas. Eye 2002; 16: 325-346.

8 Kaiserman I, Kaiserman N, Pe'er J. Long term ultrasonic follow up of choroidal naevi and their transformation to melanomas. Br J Ophthalmol 2006; 90: 994-998.

9 Singh AD, Mokashi AA, Bena JF, Jacques R, Rundle PA, Rennie IG. Small choroidal melanocytic lesions. Features predictive of growth. Ophthalmology 2006; 113: 1032-1039.

10 Straatsma BR, Landers MB, Kreiger AE, Apt L. Topography of the adult human retina. UCLA Forum Med Sci 1969; 8: 379-410.
11 Jonas JB, Gusek GC, Naumann GO. Optic disc, cup and neuroretinal rim size, configuration and correlations in normal eyes. Invest Ophthalmol Vis Sci 1988; 29: 1151-1158.

12 Li W, Judge H, Gragoudas ES, Seddon JM, Egan KM. Patterns of tumor initiation in choroidal melanoma. Cancer Res 2000; 60: 3757-3760.

13 Mosier MA. Retinal cartography. Can J Ophthalmol 1982; 17: 219-222.

14 Borodkin MJ, Thompson JT. Retinal cartography. An analysis of two-dimentional and three-dimentional mapping of the retina. Retina 1992; 12: 273-280.

15 Brown GC, Shields JA, Augsburger JJ. Amelanotic choroidal nevi. Ophthalmology 1981; 88: 1116-1121.

16 Shields CL, Furuta M, Mashayekhi A, Berman EL, Zahler JD, Hoberman DM et al. Clinical spectrum of choroidal nevi based on age at presentation in 3422 consecutive eyes. Ophthalmology 2008; 115: 546-552.

17 Shields CL, Mashayekhi A, Materin MA, Luo CK, Marr BP, Demirci $\mathrm{H}$ et al. Optical coherence tomography of choroidal nevus in 120 patients. Retina 2005; 25: 243-252.

18 Shah CP, Weis E, Lajous M, Shields JA, Shields CL. Intermittent and chronic ultraviolet light exposure and uveal melanoma. A meta-analysis. Ophthalmology 2005; 112: 1599-1607.

19 Singh AD, Rennie IG, Seregard S, Giblin M, McKenzie J. Sunlight exposure and pathogenesis of uveal melanoma. Surv Ophthalmol 2004; 49: 419-428.

20 Elwood JM, Jopson J. Melanoma and sun exposure: an overview of published studies. Int J Cancer 1997; 73: 198-203.

21 Harrison SL, MacLennan R, Speare R, Wronski I. Sun exposure and melanocytic naevi in young Australian children. Lancet 1994; 344: 1529-1532.

22 Dodd AT, Morelli J, Mokrohisky ST, Asdigian N, Byers TE, Crane LA. Melanocytic nevi and sun exposure in a cohort of Colorado children: anatomic distribution and site-specific sunburn. Cancer Epidemiol Biomarkers Prev 2007; 16: 2136-2143.

23 Shields CL, Furuta M, Mashayekhi A, Berman EL, Zahler JD, Hoberman DM et al. Visual acuity in 3422 consecutive eyes with choroidal nevus. Arch Ophthalmol 2007; 125: 1501-1507.

24 Green A. A theory of site distribution of melanomas: Queensland, Australia. Cancer Causes Control 1992; 3: 513-516.

25 Harrison SL, Buettner PG, MacLennan R. Body-site distribution of melanocytic nevi in young Australian children. Arch Dermatol 1999; 135: 47-52.

26 Whiteman DC, Brown RM, Purdie DM, Hughes M-C. Prevalence and anatomical distribution of naevi in young Queensland children. Int J Cancer 2003; 106: 930-933.

27 Krohn J, Frøystein T, Dahl O. Posterior uveal melanoma. Distribution of the sites of origin and patterns of tumour extent in the ocular fundus. Br J Ophthalmol 2008; 92: 751-756.

28 Shields CL, Shields JA, Kiratli H, De Potter P, Cater JR. Risk factors for growth and metastasis of small choroidal melanocytic lesions. Ophthalmology 1995; 102: 1351-1361.

29 Singh AD, Kalyani P, Topham A. Estimating the risk of malignant transformation of a choroidal nevus. Ophthalmology 2005; 112: 1784-1789.

30 Thiagalingam S, Wang JJ, Mitchell P. Absence of change in choroidal nevi across 5 years in an older population. Arch Ophthalmol 2004; 122: 89-93. 